\title{
BISE BEPORT
}

\section{MOBILE TELEDERMATOLOGY IN DIAGNOSIS AND MANAGEMENT OF TWO TINEA INCOGNITO CASES AT A PRIMARY HEALTH CENTER OF SEMI-URBAN KATHMANDU Shumneva Shrestha ${ }^{1}$, Randhir Sagar Yadav ${ }^{1}$, Suwash Baral ${ }^{2}$, Dwarika Prasad Shrestha ${ }^{3}$ \\ ${ }^{1}$ Tokha Chandeshwori Primary Health Center, District Public Health Office, Kathmandu, Nepal, \\ 2Department of Dermatology, Anandaban Hospital, The Leprosy Mission Nepal, Lalitpur, Nepal, \\ ${ }^{3}$ Department of Dermatology, Institute of Medicine, Tribhuvan University, Kathmandu, Nepal \\ *Correspondence to: Dr. Shumneva Shrestha, Tokha Chandeshwori Primary Health Center, District Public Health Office, Kathmandu, Nepal. Email: shumnevashrestha@gmail.com}

\begin{abstract}
Skin diseases are among leading health problems in Nepal. Fungal infections are the tenth most common reason of OPD visits in Nepal. Due to inappropriate or inadequate treatment, clinically modified tinea infections known as tinea incognito is very common. There is no dermatological care in the primary health centers. The medical officers in these centers have inadequate training to manage skin diseases, while the health workers have no training. Most dermatologists are based in tertiary hospitals and medical colleges of bigger cities. Mobile teledermatology is an innovative method to provide dermatological care to the health centers where there are no dermatologists. It is equally effective means of on-site training for the medical officers. We report two cases of tinea incognito, which were treated by a medical officer at a primary health center with the aid of mobile teledermatology consultation provided by a dermatologist.
\end{abstract}

Key words: Mobile teledermatology, Primary health center, Tinea

\section{INTRODUCTION}

Skin diseases (SDs) are common health problems. Fungal infections are the tenth most common cause of OPD consultations in Nepal. ${ }^{1}$ Tinea incognito (TI) is a superficial fungal infection that shows atypical clinical appearance because of topical steroids or calcineurin inhibitors use..$^{2,3}$ $\mathrm{TI}$ cases have increased in recent years. ${ }^{3}$ Mobile Teledermatology (MTD) provides economical, feasible and reliable dermatological consultations and follow-up treatment where specialist services are unavailable. ${ }^{4,5}$ Similarly, it can be an effective on-site training tool for medical officers (MOs) at rural settings. ${ }^{5,6}$ We herein report two TI cases which were diagnosed, followed-up and managed by a MO through MTD consultations with a dermatologist.

\section{CASE 1}

A 41-year-old male health worker presented to the OPD of Tokha Chandeshwori Primary Health Center (PHC) with itchy lesions over bilateral buttocks and lateral thigh for about three years. The rash first appeared on the left buttock. The symptoms worsened during summer and especially after riding bike. He did not complain of other skin lesion over rest of the body. There was no history of systemic or skin disorder. He applied ointment hydrocortisone intermittently on his own. During that period, the lesions spread extensively involving most of his bilateral buttocks and posterior aspect of bilateral upper thighs. Physical examination demonstrated lesions shown in figure 1A. On MTD consultation, diagnosis of $\mathrm{TI}$ was made. Routine investigations were within normal limits. $\mathrm{KOH}$ preparation showed multiple hyphae. The patient was prescribed with ointment Ketoconazole twice a day for 4 weeks, tablet Terbinafine $250 \mathrm{mg}$ per oral once a day for 2 weeks and tablet Levocetrizine $5 \mathrm{mg}$ per oral once a day if needed. His lesions recovered fully by completion of treatment (figure 1B). 


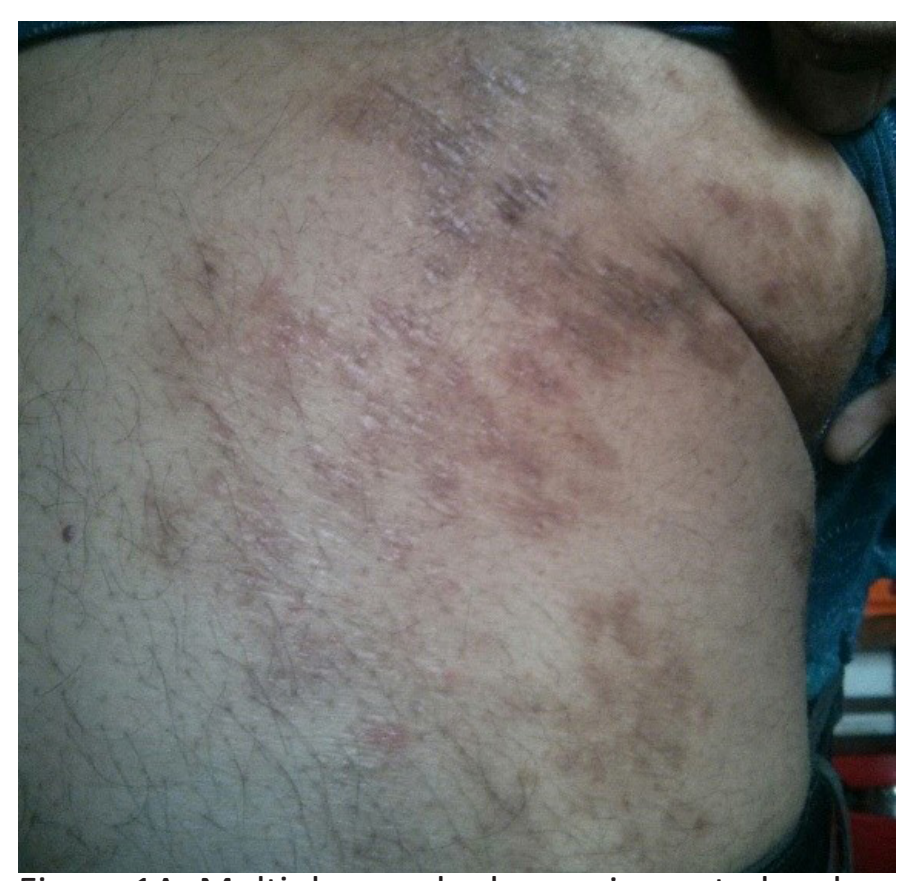

Figure 1A: Multiple annular hyperpigmented scaly lesions that coalesced into diffuse plaques at left lateral and posterior thigh and buttock, lesions before initiation of treatment

\section{CASE 2}

A 30-year-old male businessman presented to the OPD of our PHC with excessive pruritic lesion involving both groins for a month. There were no aggravating factors. He had no history of systemic or skin disease. He had applied topical corticosteroid (combination of Beclomethasone and Gentamicin) for several weeks prescribed from local pharmacy. The lesion initially improved but worsened later.

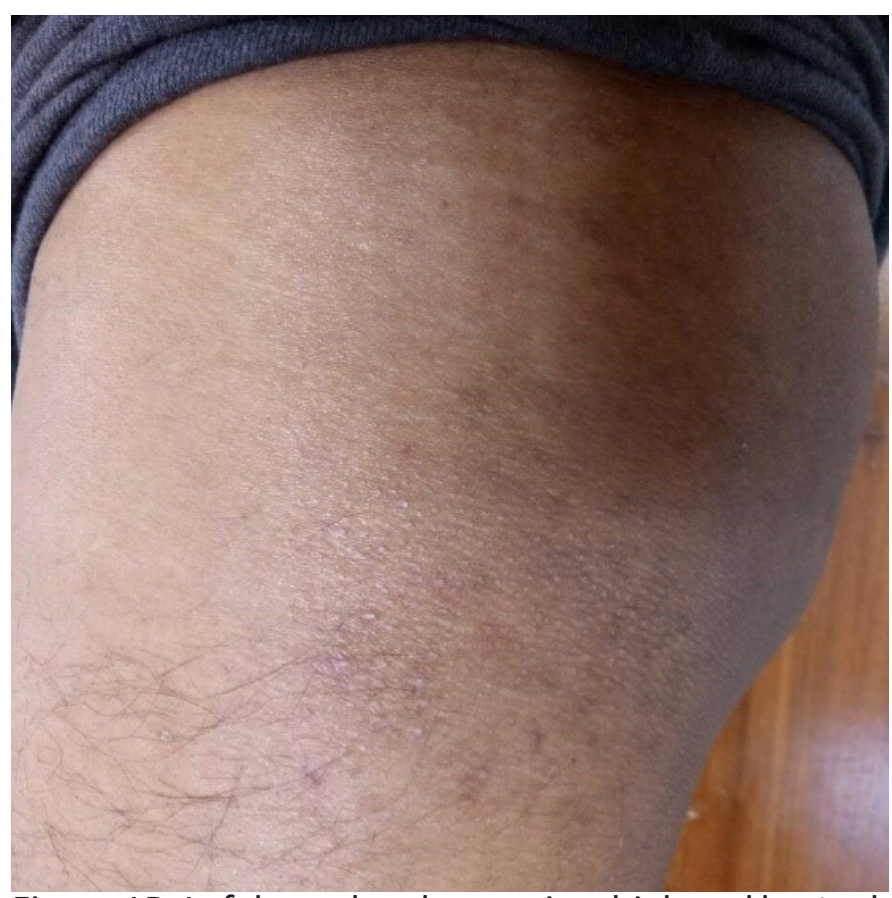

Figure 1B: Left lateral and posterior thigh and buttock after treatment completion

Physical examination revealed lesions shown in figure $2 \mathrm{~A}$ and $2 \mathrm{~B}$. On consultation with dermatologist through MTD, a diagnosis of TI was made. Routine investigations were within normal limits. $\mathrm{KOH}$ preparation showed multiple hyphae. Patient was successfully treated (figure $2 \mathrm{C}$ and 2D) with topical Ketoconazole twice a day for 6 weeks and tablet Terbinafine $250 \mathrm{mg}$ per oral once a day for 2 weeks.

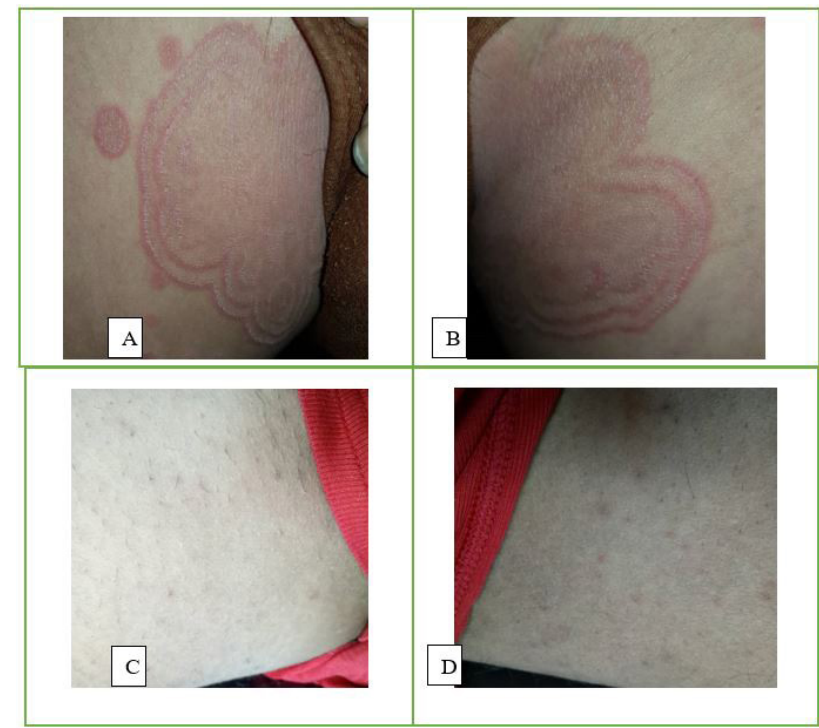

Figure 2: Multiple annular peripherally spreading erythematous scaly lesions with active borders at right groin (A) and left groin (B), lesion before initiation of treatment. C. Right groin after treatment completion. D. Left groin after treatment completion. 


\section{DISCUSSION}

Trichophyton rubrum is the most common organism causing tinea infection. Tinea are often misdiagnosed as they resemble skin lesions like rosacea, discoid lupus erythematosus, impetigo, psoriasis or eczema. TI particularly affects adults at body parts like arm, leg, buttocks and thigh. ${ }^{2}$ Similarly, it is also seen on face and trunk. ${ }^{2,3}$ Case 1 had lesion on the buttocks and thigh while case 2 had lesion at groins. Misdiagnosis of tinea and easy availability of topical steroids as over the counter (OTC) drug to treat tinea infections have supplemented in rising $\mathrm{Tl}$ cases. Most of topical steroid use are found prescribed by pharmacist or paramedicals. Majority of OTC topical steroids belong to potent and very potent groups. ${ }^{7}$ Case 1 had selfdiagnosed his lesion as dermatitis and used topical steroid. Case 2 got topical steroid prescribed from local pharmacy. Similarly, misuse of topical steroids is another worrisome effect on dermatological practice. ${ }^{7}$ Both the cases were misdiagnosed initially and misused topical steroid. Both cases were diagnosed by dermatologists through MTD consultations and $\mathrm{KOH}$ preparation confirmed the diagnosis. Potent topical steroid increases number of hyphae giving a different appearance. TI should be suspected if the skin lesion is treated with steroid but doesn't respond to it. ${ }^{3}$ Case 2 noticed initially regression of lesion that flared up later on topical steroid while case 1 noticed gradual progression of lesions with time. Besides topical steroid use, it was found that $40 \% \mathrm{TI}$ cases received systemic steroid for non-dermatologic conditions. ${ }^{2}$ Topical antifungals are the treatment of choice of $\mathrm{TI}^{8}{ }^{8}$ Oral antifungals are advised in cases of extensive lesions or topical antifungal resistant lesions. ${ }^{9}$ Generally 2 weeks of therapy is indicated for dermatophytoses whereas the clinical outcome and fungal response determine the total duration of therapy. ${ }^{10}$ Both cases required oral antifungals because of the misuse of topical steroid which flared up the disease. It took 4 and 6 weeks for complete resolution in case 1 and 2 respectively.

In Nepal, there is very limited number of dermatologists to care for huge number of SDs. Additionally, most of them practice in tertiary hospitals and medical colleges whereas $85 \%$ of people reside in rural Nepal where MOs and paramedicals are the primary service providers. Thus, majority of Nepalese are devoid of dermatological care. ${ }^{4}$ In such context, MTD can be an efficient tool to provide specialist dermatological care to the underserved population. ${ }^{5}$ MOs have limited dermatological training while health workers or paramedicals have no training. ${ }^{4,6}$ Case 1 was himself a health worker and case 2 was prescribed topical steroid from a local pharmacy. But none of them had adequate knowledge on managing tinea. Furthermore, it is much harder for them to diagnose and manage TI which at times are diagnostic challenge for specialists due to atypical clinical presentation. MOs had difficulty in diagnosing these cases which were eventually diagnosed by dermatologist's consultations on MTD. Similarly, studies have shown MTD as an on-site educational and training support tool for MOs and junior doctors working at those health facilities. ${ }^{5,6}$ MTD was found as reliable, useful and cost effective means of dermatological consultations with a high concordance rate in respect to face-to-face consultations. ${ }^{4,5}$ Thus, MTD can be an effective way for diagnosis, follow-up and management of SDs like TI at PHCs.

\section{CONCLUSION}

TI cases are rising in parts where dermatologists are not available. Topical steroids are available as OTC drug at majority of pharmacies of Nepal and are being misused. MTD can be the most useful tool to provide specialist dermatological care to rural and semi-urban population. It can also help to train and educate MOs on SDs and rational use of topical steroids.

\section{REFERENCES}

1. Department of Health Services. Annual Report 2073/74 (2016/17), Department of Health Services, Ministry of Health and Population, Kathmandu, 2018. Available at http://dohs. gov.np/wp-content/uploads/2018/04/Annual_ Report_2073-74.pdf

2. Romano C, Maritati E, Gianni C. Tinea incognito in Italy: a 15-year survey. Mycoses. 2006;49(5):3837. [PubMed]

3. Del Boz J, Crespo V, Rivas-Ruiz F, De Troya M. Tinea incog $\neg$ nito in children: 54 cases. Mycoses. 2011;54:254-8. [PubMed] 
4. Shrestha DP, Suwash B, Gurung D, Uprety A, Bhattarai S, Rosdahl I. Mobile teledermatology for rural Nepal: Dermatologic care using mobile phones in a primary health care centre. Journal of Institute of Medicine. 2016;38(1):7-10. [Full Text]

5. Tran K, Ayad M, Weinberg J, Cherng A, Chowdhuty $\mathrm{M}$, Monir S, et al. Mobile teledermantology in the developing word: Implications of a feasibility study on 30 Egyptian patients with common skin disorders. J Am Acad Dermatol. 2011;64(2):3029. [PubMed]

6. Shrestha DP, Baral S, Shahi PV, Rosdahl I. Mobile teledermatology for Dermatologic care in rural Nepal: Dermatology training of medical officers at primary health care centres. Journal of
Institute of Medicine. 2017;39(2):3-7. [Full Text]

7. Dey VK. Misuse of topical corticosteroids: A clinical study of adverse effects. Indian Dermatol Online J. 2014;5(4):436-40. [PubMed]

8. Lesniak R. Tinea incognito. Dermatol Nurs. 2008;20(5):403-4. [PubMed]

9. Lange $M$, Jasiel-Walikowska E, Nowicki R, Bykowska B. Tinea incognito due to Trichophyton mentagrophytes. Mycoses. 2010;53(5):455-7. [PubMed]

10. Guenova E, Hoetzenecker W, Schaller M, et al. Tinea incognito hidden under apparently treatment-resistant pemphigus foliaceus. Acta Derm Venereol. 2008;88(3):276-7. [PubMed] 